\title{
Is Brain MRI Needed in Diagnostic Evaluation of Mild Intellectual Disability?
}

\author{
Miro Pekka Jussila1® Päivi Olsén ${ }^{2}$ Jaakko Niinimäki ${ }^{1}$ Maria Suo-Palosaari ${ }^{1}$ \\ ${ }^{1}$ Department of Diagnostic Radiology, Oulu University Hospital and
Research Unit of Medical Imaging, Physics and Technology, Oulu
University Hospital and University of Oulu, Oulu, Finland
2 Department of Pediatrics and Adolescence, Oulu University Hospital
and PEDEGO Research Unit, Oulu University Hospital and University
of Oulu, Oulu, Finland \\ Address for correspondence Maria Suo-Palosaari, MD, PhD, \\ Department of Diagnostic Radiology, Oulu University Hospital and \\ Research Unit of Medical Imaging, Physics and Technology, Oulu \\ University Hospital and University of Oulu, Kajaanintie 50, PO Box 50, \\ 90029 OYS, Oulu, Finland (e-mail: maria.suo-palosaari@ppshp.fi).
}

Neuropediatrics 2021;52:27-33.

\begin{abstract}
Keywords

- brain

- MRI

- intellectual disability

- children

- etiology

Aim The purpose of our study was to suggest an imaging strategy and guidelines for the selection of the children with mild intellectual disability (ID) for magnetic resonance imaging (MRI), to avoid unnecessary imaging.

Methods The brain MRIs and patient reports of 471 children were reviewed for the imaging findings and ID severity. The correlation between the clinical and brain MRI findings was analyzed in the 305 children with mild ID.

Results Thirty-eight (12.5\%) of the children with mild ID had significant abnormal brain MRI findings. Thirty-five of these had other neurological symptoms or diseases in addition to ID, which were an indication for brain MRI. In the logistic regression analysis, seizures (in patients without an epilepsy diagnosis), epilepsy, movement disorders, dysmorphia, encephalitis, traumatic brain injury, and abnormal head size were statistically significant symptoms or comorbidities associated with abnormal MRI findings. Only three children (1.0\%) with mild ID had a significant MRI finding without any other clinical symptoms or disease.

Conclusion Routine MRI in children with mild ID without specific neurological symptoms, dysmorphic features, or related diseases is not suggested for revealing an etiology of mild ID. Since children with ID usually need to be sedated for MRI, routine imaging in the diagnostic evaluation of mild ID should be carefully considered. Clinical examination, other symptoms, and related diseases should be carefully assessed to decide the need for MRI.
\end{abstract}

\section{Introduction}

Intellectual disability (ID) concerns early onset of cognitive impairment and limitations of skills in several functional areas. ${ }^{1}$ The diagnosis is defined by significant limitations in intellectual functioning evaluated by valid assessment of intelligence. Additional ID criteria include significant deficits in functional and adaptive skills and onset before the age of 18 years. ${ }^{2}$ Intelligence quotient (IQ) testing, however, is not generally applicable for children under 3 years of age, and

received

April 26, 2020

accepted

July 28,2020

published online

October 27, 2020 scoring of developmental delay is more reliable in older age groups. ${ }^{3}$ The estimated prevalence of ID varies between 1 and $3 \%$ and is lower for more severe ID (IQ $<50$, prevalence $<0.5 \%$ ) than for mild ID (IQ $70-50)^{4,5}$ In the nationwide register-based study, prevalence of mild ID was $0.52 \%{ }^{6}$

Although clinical assessment is necessary to evaluate an underlying etiology of ID, the role of neuroimaging in diagnostics remains unknown. The goals of neuroimaging in ID are to find a lead to the etiological diagnosis to counsel children with ID and their families adequately, and to detect (c) 2020. Thieme. All rights reserved. Georg Thieme Verlag KG, Rüdigerstraße 14,

70469 Stuttgart, Germany
DOI https://doi.org/ 10.1055/s-0040-1716902. ISSN $0174-304 X$. 
anomalies or diseases that would have consequences for the patient care. Some practitioners conduct brain magnetic resonance imaging (MRI) of children with ID in the presence of certain symptoms or comorbidities, while others recommend MRI for all children with unexplained neurodevelopmental delay. ${ }^{7-11}$ In a previous study, MRI was recommended only after other tests (standardized psychometric tests, clinical examination, electroencephalogram, and laboratory tests [including karyotyping and fragile- $\mathrm{X}$ syndrome]) if the etiology of ID remained unknown. ${ }^{12}$ Based on the literature, routine imaging studies are rarely suggested to be useful when identifying the etiology of ID or developmental delay. ${ }^{7,8,10,12,13}$

In Finland, every child with ID undergoes MRI at the time of diagnosis regardless of the grade of ID or comorbidities. However, based on the earlier literature, it is known that significant MRI findings are not found in most cases with mild ID. ${ }^{14}$ Therefore, we focused on the brain MRI findings of the mild form of ID to suggest an imaging strategy and guidelines for the selection of children with mild ID for MRI, to avoid unnecessary imaging.

\section{Methods}

\section{Study Design and Population}

This study included a retrospective regional population of 0 to16-year-old children diagnosed with ID during the years 1999 to 2018 in the Northern Ostrobothnia Hospital District in Finland. Their diagnosis was conducted at Department of Pediatric Neurology of Oulu University Hospital, a tertiary referral hospital.

The children, we focused on in this study, had mild ID diagnosed by psychometric tests and by a clinician's evaluation. A pediatric neurologist (P.O., 20 years of experience in pediatric neurology) and a final-year medical student (M.-P. J.) reviewed the patient reports for neurological symptoms and related diseases, IQ assessments, and laboratory tests. The ID severity was classified with an International Classification of Diseases (ICD)-10th revision code system (F70-79; ICD-10, World Health Organization [WHO]). ${ }^{15}$ Neurological symptoms and factors estimated to correlate with the etiology of ID or other comorbidities were collected and analyzed.

Prior to the initiation of the study, a sample size was calculated: the population estimate rate of children with ID in this tertiary referral hospital's area was 1,300 . The assumption of a significant MRI finding to be found was in $8 \%$ of cases, with a maximum deviation of $\pm 2 \%$ and a confidence level of $95 \%$. Therefore, 458 was estimated to be the sample size for children with ID. The sample selection was 471 of the 0 - to 16-year-old children with ID, who were born between the $1 \mathrm{st}^{\mathrm{t}}$ and 19th days of every month between 1990 and 2017. Of them, 305 had mild ID. - Fig. 1 shows the flowchart of the study population selection.

\section{Ethical Approval}

All procedures performed in the studies involving human participants were in accordance with the ethical standards of Oulu University Hospital and/or the national research com- mittee and with the 1964 Helsinki Declaration and its later amendments or comparable ethical standards. For this type of study formal consent of the participants is not required.

\section{MRI Procedure}

All the children with ID had been imaged by brain MRI. The MRI scans were performed at Oulu University Hospital, Kainuu Central Hospital, Länsi-Pohja Central Hospital, Lapland Central Hospital, or Ostrobothnia Central Hospital between 1996 and 2018. They were acquired with 1.5-T MRI scanners. The imaging protocol included at least T1and T2-weighted, and T2-weighted fluid-attenuated inversion recovery sequences (slice thickness was 4-6 $\mathrm{mm}$ and a gap of $0.5-1.5 \mathrm{~mm}$ ). The protocol included sequences in three planes. The children had been sedated during imaging when necessary. The brain MRI abnormalities were reevaluated and classified by a pediatric radiologist (M.S.-P., 9 years of experience in pediatric neuroradiology), who was blinded for the specific clinical features of the children beside the presence of neurodevelopmental disorders. The significant imaging findings were defined as abnormalities supporting the etiological diagnosis for ID.

\section{Statistical Analysis}

To evaluate the correlation of neurological symptoms and clinical findings to the imaging findings, multivariate logistic regression analysis was used. In the logistic regression analysis, the forward stepwise selection method was used to remove insignificant variables from the analysis. The Chisquared test was used to determine whether there was a difference in symptoms or imaging findings between male and female patients. To compare the prevalence of the imaging findings between different grades of ID, the Chisquared test was used. The Chi-squared test was also used to compare the prevalence of symptoms in two groups (patients with and without imaging findings). The patients were divided into four age groups (0-2, 3-6, 7-10, and over 10 years), due to the assumption of different etiologies in particular age groups. The Chi-squared test was used to compare symptoms and imaging findings between age groups. For all tests, a significance level of $<0.05$ was used. All data were analyzed using IBM SPSS Statistics 25.0 software (IBM Corp., Armonk, New York, United States).

\section{Results}

Among 126 (26.9\%) of 471 children with ID, there were significant imaging findings. Patients were divided into groups based on ICD-10 classification of ID (mild, moderate to severe, and unspecified). In mild ID cases, $12.5 \%$ of 305 children had a significant imaging finding. Children with moderate-to-severe ID ( $55.2 \%$ of 134 children, $p<0.001$ ) and unspecified ID $(43.8 \%$ of 32 children, $p<0.001)$ had more imaging findings than children with mild ID.

During the years 1999 to 2018, 305 patients of 471 had been diagnosed with mild ID (-Fig. 1). Of them, 98 were female and 207 male. The mean age of the children at diagnosis was $6.9 \pm 3.4$ years. A significant imaging finding 


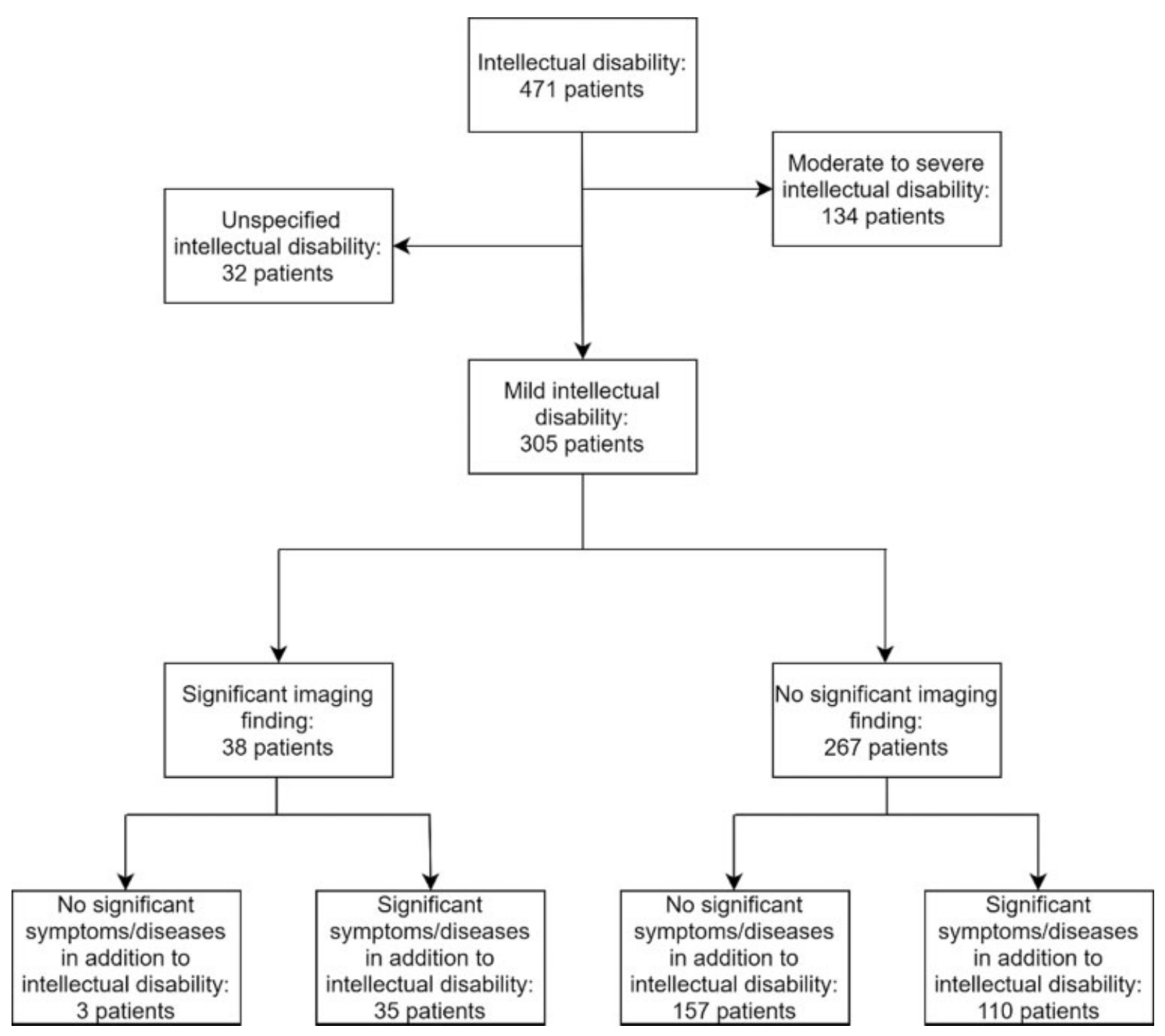

Fig. 1 Study population flowchart.

that, most probably, correlated with ID or with neurological symptoms or diseases was found in 38 (12.5\%) of these children. In the other cases, the brain MRIs were normal or with incidental findings that did not correlate with ID or related comorbidities. The significant abnormal imaging findings are listed in -Table $\mathbf{1}$.

Only three of the children (1.0\%) with mild ID had a significant imaging finding but no neurological symptoms or other diseases in addition to ID. The imaging findings of these three children were frontal pachygyria, syntelencephaly, and hypoplastic right cerebellar hemisphere. Two children were 9 years old and one child was 6 years old at the time of the ID diagnosis.

The symptoms and comorbidities are listed in - Table 2 . The most common were epilepsy, dysmorphic features, premature birth, hypotonia, and movement disorders ( - Table 2). In some cases, the above-mentioned neurological symptoms occurred only after the imaging had been performed, but these children were included in the analyses. No statistically significant differences of significant imaging were found between the four age groups (0-2, 3-6, 7-10, and over 10 years). However, the patients in the youngest age group had significantly more symptoms ( 87.5 vs. $47.5 \%$ of all the children with mild ID; $p<0.001$ ). In the other age groups, $44.3 \%$ (3-6 years), 36.1\% (710 years), and $64.6 \%$ (over 10 years) of the children with mild ID had neurological symptoms or clinical findings.

Of the children with neurological symptoms or clinical findings $(n=145), 24.1 \%(n=35)$ had significant imaging findings in MRI. Epilepsy $(p=0.011)$, traumatic brain injury $(p=0.003)$, and paralysis or cerebral palsy $(p=0.001)$ were more common in patients who had imaging findings. There were no statistically significant differences in other symptoms or clinical findings when comparing patients with and without an imaging finding (-Table 2 ).

To evaluate the correlation of multiple symptoms or clinical findings to significant MRI findings, logistic regression analysis was also used. The variables used are described in - Table 3 . All the children with mild ID $(n=305)$ were included in the regression analysis. Insignificant variables were removed from the regression model (forward step-wise selection was used). In the logistic regression analysis, seizures (without an epilepsy diagnosis), epilepsy, movement disorders, 
Table 1 Significant imaging findings of 38 children with mild intellectual disability

\begin{tabular}{|l|l|l|}
\hline Imaging finding & $\boldsymbol{n}$ & $\%$ \\
\hline Corpus callosal anomalies & 14 & 36.8 \\
\hline Ventricular dilatation & 13 & 34.2 \\
\hline Abnormal gyration & 10 & 26.3 \\
\hline Cerebral atrophy & 9 & 23.7 \\
\hline Intracranial hemorrhage & 5 & 13.2 \\
\hline Delayed myelination & 5 & 13.2 \\
\hline Leukomalacia & 4 & 10.5 \\
\hline Diffuse axonal injury & 3 & 7.9 \\
\hline Nodular heterotopia & 3 & 7.9 \\
\hline Cerebellar hypoplasia & 3 & 7.9 \\
\hline Abnormal white matter signal & 3 & 7.9 \\
\hline $\begin{array}{l}\text { Central nervous system infection, } \\
\text { status post }\end{array}$ & 2 & 5.3 \\
\hline Syntelencephaly & 1 & 2.6 \\
\hline Meningomyelocele (Chiari II) & 1 & 2.6 \\
\hline Tuberous sclerosis & 1 & 2.6 \\
\hline Total & $77^{\text {a }}$ & \\
\hline
\end{tabular}

${ }^{\text {aS }}$ ome children had more than one imaging finding. dysmorphia, encephalitis, traumatic brain injury, and abnormal head size were the variables that significantly increased the likelihood of a significant imaging finding. All children with cerebral palsy or paralysis had a significant imaging finding. Cerebral palsy was not a statistically significant prognostic factor because there were only a few patients. In addition to clinical signs and symptoms, age and gender were also included in the regression model, but they did not increase the risk of an abnormal MRI finding. There was no statistically significant difference in symptoms or imaging findings between males and females.

In the end stage of the analysis, we checked if genetic etiologies were found in the children with mild ID. In 48 of the 305 children with mild ID, a genetic error was found; of them, 13 children also had an imaging finding. These 13 children had other symptoms or diseases in addition to ID. Genetic errors and imaging findings of those 13 patients are presented in - Table 4. In majority of these cases, the imaging findings were not specific to any known genetic etiology. However, imaging findings in these cases were significant and would have led to further etiological investigations.

\section{Discussion}

To evaluate the etiology of ID, many children are referred for MRI. ${ }^{16}$ All major recent studies do not recommend routine brain MRI in ID or developmental delay in the absence of

Table 2 Children with mild intellectual disability with symptoms/clinical findings $(n=145)$

\begin{tabular}{|l|l|l|l|l|}
\hline \multirow{2}{*}{ Symptom or clinical finding } & \multicolumn{2}{l|}{$\begin{array}{l}\text { Symptoms/clinical } \\
\text { findings, no imaging } \\
\text { findings }(\boldsymbol{n}=110)\end{array}$} & \multicolumn{2}{l|}{$\begin{array}{l}\text { Symptoms/clinical findings } \\
\text { and imaging findings }(\boldsymbol{n}=35)\end{array}$} \\
\cline { 2 - 5 } & $\boldsymbol{n}$ & $\%$ & $n$ & $\%$ \\
\hline Dysmorphia & 39 & 35.5 & 9 & 25.7 \\
\hline Epilepsy & 15 & 13.6 & 12 & 34.3 \\
\hline Preterm birth & 16 & 14.5 & 4 & 11.4 \\
\hline Hypotonia & 15 & 13.6 & 5 & 14.3 \\
\hline Slow growth & 12 & 10.9 & 2 & 5.7 \\
\hline Movement disorder & 7 & 6.4 & 6 & 17.1 \\
\hline Seizure (not epilepsy) & 5 & 4.5 & 3 & 8.6 \\
\hline Other abnormality in neurological status & 5 & 4.5 & 1 & 2.9 \\
\hline Abnormal head size & 4 & 3.6 & 2 & 5.7 \\
\hline Asphyxia & 3 & 2.7 & 2 & 5.7 \\
\hline Metabolic disease & 3 & 2.7 & 1 & 2.9 \\
\hline Intrauterine growth restriction & 3 & 2.7 & 1 & 2.9 \\
\hline Muscle disease & 2 & 1.8 & 0 & 0 \\
\hline Traumatic brain injury & 1 & 0.9 & 5 & 14.3 \\
\hline Central nervous system infection, status post & 1 & 0.9 & 2 & 5.7 \\
\hline Acute lymphocytic leukemia & 1 & 0.9 & 0 & 0 \\
\hline Paralysis or cerebral palsy & 0 & 0 & 5 & 14.3 \\
\hline
\end{tabular}


Table 3 Logistic regression analysis of children with mild intellectual disability

\begin{tabular}{|l|l|l|l|l|l|l|}
\hline & \multicolumn{2}{l|}{ Univariate } & \multicolumn{2}{l|}{ Multivariate } \\
\hline Variables & OR & $95 \% \mathrm{Cl}$ & $p$-Value & OR & $95 \% \mathrm{Cl}$ & $p$-Value \\
\hline Seizure (not epileptic) & 4.49 & $1.03-19.62$ & 0.046 & 7.93 & $1.43-43.89$ & 0.018 \\
\hline Epilepsy & 7.75 & $3.28-18.32$ & $<0.001$ & 8.85 & $3.07-25.54$ & $<0.001$ \\
\hline Traumatic brain injury & 40.30 & $4.57-355.57$ & 0.001 & 80.82 & $7.88-829.24$ & $<0.001$ \\
\hline $\begin{array}{l}\text { Central nervous system } \\
\text { infection, status post }\end{array}$ & 14.78 & $1.31-167.12$ & 0.030 & 31.07 & $2.20-439.02$ & 0.011 \\
\hline Movement disorders & 6.96 & $2.20-22.01$ & 0.001 & 11.85 & $3.13-44.89$ & $<0.001$ \\
\hline Dysmorphia & 1.81 & $0.80-4.13$ & 0.155 & 2.74 & $1.05-7.13$ & 0.040 \\
\hline Abnormal head size & 3.65 & $0.65-20.66$ & 0.143 & 7.07 & $1.04-48.09$ & 0.046 \\
\hline Paralysis $^{\text {a }}$ & $1.31 \mathrm{E}+10$ & $0.000-$ & 0.999 & $1.18 \mathrm{E}+10$ & 0.00 & 0.999 \\
\hline
\end{tabular}

Abbreviations: $\mathrm{Cl}$, confidence interval; OR, odds ratio.

an the logistic regression analysis, paralysis had a very high odds ratio and the $p$-value was 0.999 . This is explained by the fact that all patients with paralysis had imaging findings.

Table 4 Genetic etiology with associated imaging findings in 13 children

\begin{tabular}{|c|c|c|}
\hline & Genetic etiology & Imaging finding \\
\hline 1 & Chromosome deletion 2q33.3-34 (1.3 Mb) & Delayed myelination, CC hypoplasia \\
\hline 2 & NHLRC2-gene mutation (FINCA disease) & Brain atrophy and thin $\mathrm{CC}$ \\
\hline 3 & Chromosome 7 microdeletion (2.37 Mb) & Dandy-Walker variant \\
\hline 4 & IQSEC2-gene hemizygotic mutation & Brain atrophy, delayed myelination, thin CC \\
\hline 5 & CRADD-gene homozygotic mutation & Frontal pachygyria, temporal polymicrogyria \\
\hline 6 & $\begin{array}{l}\text { Two chromosomal microdeletions (del (2) } \\
(\text { p22.1p21)) and (del (6) (q27q27)) }\end{array}$ & Brain atrophy, thin CC \\
\hline 7 & $\begin{array}{l}\text { Chromosome } 6 \mathrm{p} 25 \text { deletion }(2.3 \mathrm{Mb}) \\
\text { and 9p24 duplication ( } 6.7 \mathrm{Mb})\end{array}$ & Hydrocephalus, confluent white matter abnormalities, thin CC \\
\hline 8 & MED12-gene mutation (Lujan-Fryns syndrome) & $\begin{array}{l}\text { Ventricular dilatation, macrocephaly, CC agenesis, } \\
\text { hypoplasia of nervus opticus }\end{array}$ \\
\hline 9 & $\begin{array}{l}\text { Orofacial-digital syndrome type } 1 \\
\text { (Papillon-Leage-Psaume syndrome) }\end{array}$ & $\begin{array}{l}\text { Ventricular dilatation, frontal and occipital polymicrogyria, } \\
\text { nodular heterotopia, CC agenesis }\end{array}$ \\
\hline 10 & $\begin{array}{l}\text { Triple-A syndrome homozygotic } \\
\text { gene mutation }\end{array}$ & $\begin{array}{l}\text { Ventricular dilatation, atrophy and signal } \\
\text { abnormalities of basal ganglia }\end{array}$ \\
\hline 11 & $\begin{array}{l}\text { Chromosome } 10 \text { microduplication } \\
\text { 10p15.1-15.3 ( } 6.5 \mathrm{Mb}) \\
\text { and chromosome } 18 \text { microdeletion } \\
18 \mathrm{q} 22.3-\mathrm{q} 23(6.9 \mathrm{Mb})\end{array}$ & Delayed myelination \\
\hline 12 & Chromosome 10 microdeletion & Ventricular dilatation, delayed myelination, thin CC \\
\hline 13 & Tuberous sclerosis (based on clinical findings) & $\begin{array}{l}\text { Cortical/subcortical tubers and subependymal nodules, } \\
\text { cerebellar calcifications }\end{array}$ \\
\hline
\end{tabular}

Abbreviations: CC, corpus callosum; FINCA, fibrosis, neurodegeneration and cerebral angiomatosis.

other symptoms or comorbidities. ${ }^{7,14,17}$ In a clinical report from the American Academy of Pediatrics, the authors stated that brain MRI should be done if patients have microcephaly, macrocephaly, or abnormal findings on neurologic examination. ${ }^{17}$ An etiology has been found more often in severe than in the mild form of ID by neuroimaging. ${ }^{10}$ In a previous study, IQ was shown to correlate negatively with the total abnormality score in relation to brain anomalies in subjects with ID. ${ }^{18}$ Our study focused on imaging findings of children with mild ID.
A retrospective report concerning children with developmental delay reported structural brain abnormalities in 54\% of the 132 patients; $27 \%$ of the abnormalities were considered clinically significant. ${ }^{19}$ However, a later study from the same research group showed a lower rate of brain abnormalities, $19 \%$ as against $27 \%$ in the earlier study, suggesting that less severely delayed children were included in the study group. ${ }^{16}$ Abnormal brain MRI findings contributing to the etiology of the developmental delay were found in less than $9 \%$ of 325 patients with developmental delay $(\mathrm{IQ}<70) .{ }^{20} \mathrm{~A}$ 
review that summarized 18 cross-sectional and 11 casecontrol studies of neuroimaging abnormalities in children with ID/developmental delay and no other symptoms found abnormal MRI findings in 38\% of those children, and $7.9 \%$ of those abnormalities led to an etiological diagnosis of ID/ developmental delay. ${ }^{7}$ Moreover, abnormal brain MRI findings have been found to be more common in children with mild-to-severe ID (63\%) than in the control group (30\%), while many of those imaging findings seemed to be nonspecific. $^{21}$ The populations of the above-mentioned studies differ significantly from each other, which makes a solid comparison of the results very difficult.

In this study, neuroimaging yielded the diagnosis or yielded a finding that had consequences for the patient care in 38 (12.5\%) of the children with mild ID. Thirty-five of these 38 children had also other neurological symptoms or diseases in addition to ID that would have led to neuroimaging even in the absence of ID. Engbers et al demonstrated that in a cohort of children with developmental delay (183 mild and 227 severe), 30.7\% had brain MRI abnormalities, of which $5.4 \%$ led to an etiology of developmental delay. In accordance with our results, only $1.9 \%$ of those children had a diagnostic MRI finding and no other symptoms. ${ }^{8}$ Furthermore, a study by Decobert et al found a significant imaging finding suggesting an etiology of ID on 5\% of MR scans, but these children had moderate-to-severe ID and almost all had other neurological symptoms. ${ }^{12}$ In our study, the neuroimaging findings of the three children without other symptoms were pachygyria, syntelencephaly, and hypoplastic cerebellar hemisphere. Similarly, pachygyria has been previously demonstrated in a child with developmental delay without seizures or other neurological symptoms. ${ }^{22}$

In a previous study, the administration of MRI contrast agent was not helpful when only determining an etiology of the developmental delay. When developmental delay was a secondary concern, only $11.1 \%$ of cases were administration of contrast agent considered helpful in making the radiologic diagnosis. However, in none of the cases was gadolinium contrast agent essential for diagnosis. ${ }^{23}$ Our MRI protocol used for the diagnosis of ID does not include contrast-enhanced sequences unless some abnormal findings found during imaging would induce administration of enhancement.

The most common findings associated with ID in our study were corpus callosum anomalies, pachygyria, polymicrogyria, ventricular dilation, and cerebral atrophy, similar to findings in several previous studies in children with ID. ${ }^{7,8,10,18}$ Ventricular dilation is a common finding in children with developmental delay (12-48\%) $)^{10,12,24}$ but also in control patients $(20 \%)^{18,25}$ Corpus callosum abnormalities have been reported in 14 to $46 \%$ of children with ID, ${ }^{10,12,18}$ compared with $5.0 \%$ in controls. ${ }^{18} \mathrm{~A}$ meta-analysis of incidental brain MRI findings in children showed that corpus callosum anomalies are very rare (0.7\%), and no complete agenesis has been reported in healthy children. ${ }^{26}$ In accordance with Decobert et al, we suggest that the specific diagnostic value of these subtle neuroimaging findings concerning ventricular dilation and corpus callosum anomalies is low. ${ }^{12}$
A prior study demonstrated that neuroimaging performed for a specific indication was more than three times as likely to result in an etiological diagnosis than imaging done on a screening basis (41.2 vs. $13.9 \%) .{ }^{27}$ In our study, some neurological symptoms and comorbidities of the children with mild ID seemed to be better prognostic factors for a significant imaging finding. These symptoms were movement disorders (e.g., ataxia), seizures (without an epilepsy diagnosis), epilepsy, head size abnormality, traumatic brain injury, encephalitis, and dysmorphia. Cerebral palsy or paralysis seemed also to be a significant prognostic factor, because in our study, none of the children with cerebral palsy or paralysis had normal brain MRIs. In previous studies, pyramidal disorders, movement disorders, and head size abnormality have also been shown to correlate to brain MRI abnormalities. ${ }^{8,12}$ Reid et al demonstrated that only $7.9 \%$ of children with ID and cerebral palsy had normal brain MRIs. ${ }^{28}$ Encephalitis, epilepsy, and head trauma, as one might except, were significant factors predicting imaging findings, and these symptoms are solely an indication for neuroimaging. In the study by Momen et $\mathrm{al}^{29}$ younger children who had developmental delay tended to have more abnormal imaging findings compared with older children. However, those imaging findings were not often significant and were not seen on later follow-up MRIs due to brain maturation. ${ }^{29}$ This was not the case in our study, but the number of very young patients was low due to the difficulty in judging mild ID in very young patients.

\section{Limitations}

There were some limitations in our study. One was the retrospective nature of this research. Due to that fact, some MRI scans have been taken before the diagnosis of ID, but we still included them in the study. The imaging of the studied children had partly been done before the advanced molecular genetic methods were available, so the possible exact genetic diagnosis can be missing in some patients. Also, the lack of a control group is a shortage.

\section{Conclusion}

Unnecessary routine brain MRI in mild ID causes psychological and physical burdens for children and their parents. Children with ID usually require sedation or general anesthesia during MRI, which is always a risk. ${ }^{30}$ If a child has mild ID and no other neurological symptoms or related diseases, significant neuroimaging findings are not found in most cases. However, normal brain MRI finding may occasionally be helpful when excluding some differential diagnostic possibilities. Furthermore, parents of the children with ID may feel relieved if the brain MRI is normal. Nevertheless, imaging studies should only be done if there is a justifiable indication. The benefits of the imaging studies should outweigh the potential disadvantages. We suggest that clinicians consider justification for brain MRI carefully after having proper anamnesis, clinical examination, and laboratory testing. 


\section{Conflict of Interest}

None declared.

\section{Acknowledgments}

This work was supported by the Finnish Cultural Foundation, Finland, the Radiological Society of Finland, and the Arvo and Lea Ylppö Foundation, Finland.

\section{References}

1 Pratt HD, Greydanus DE. Intellectual disability (mental retardation) in children and adolescents. Prim Care 2007;34(02):375-386 , abstract ix

2 Black DW, Grant JE. DSM-5 Guidebook: the Essential Companion to the Diagnostic and Statistical Manual of Mental Disorders, 5th ed. Washington, DC: American Psychiatric Publishing; 2014

3 Shevell M, Ashwal S, Donley D. Quality Standards Subcommittee of the American Academy of Neurology Practice Committee of the Child Neurology Society, et al; Practice parameter: evaluation of the child with global developmental delay: report of the Quality Standards Subcommittee of the American Academy of Neurology and The Practice Committee of the Child Neurology Society. Neurology 2003;60(03):367-380

4 Westerinen H, Kaski M, Virta L, Almqvist F, livanainen M. Prevalence of intellectual disability: a comprehensive study based on national registers. J Intellect Disabil Res 2007;51(Pt 9):715-725

5 Ropers HH. Genetics of early onset cognitive impairment. Annu Rev Genomics Hum Genet 2010;11:161-187

6 Westerinen H, Kaski M, Virta LJ, Kautiainen H, Pitkälä KH, Iivanainen $\mathrm{M}$. The nationwide register-based prevalence of intellectual disability during childhood and adolescence. J Intellect Disabil Res 2017;61(08):802-809

7 Murias K, Moir A, Myers KA, Liu I, Wei XC. Systematic review of MRI findings in children with developmental delay or cognitive impairment. Brain Dev 2017;39(08):644-655

8 Engbers HM, Nievelstein RAJ, Gooskens RHJM, et al. The clinical utility of MRI in patients with neurodevelopmental disorders of unknown origin. Eur J Neurol 2010;17(06):815-822

9 Mithyantha R, Kneen R, McCann E, Gladstone M. Current evidence-based recommendations on investigating children with global developmental delay. Arch Dis Child 2017;102 (11):1071-1076

10 Soto-Ares G, Joyes B, Lemaître MP, Vallée L, Pruvo JP. MRI in children with mental retardation. Pediatr Radiol 2003;33(05): 334-345

11 Rodriguez DP, Poussaint TY. Neuroimaging of the child with developmental delay. Top Magn Reson Imaging 2007;18(01): 75-92

12 Decobert F, Grabar S, Merzoug V, et al. Unexplained mental retardation: is brain MRI useful? Pediatr Radiol 2005;35(06): 587-596

13 Ali AS, Syed NP, Murthy GSN, et al. Magnetic resonance imaging (MRI) evaluation of developmental delay in pediatric patients. J Clin Diagn Res 2015;9(01):TC21-TC24
14 van Karnebeek CD, Jansweijer MC, Leenders AG, Offringa M, Hennekam RC. Diagnostic investigations in individuals with mental retardation: a systematic literature review of their usefulness. Eur J Hum Genet 2005;13(01):6-25

15 World Health Organization. International Classification of Diseases (ICD). . Available at: https://www.who.int/classifications/ icd/en/. Accessed March 13, 2020

16 Griffiths PD, Batty R, Warren D, et al. The use of MR imaging and spectroscopy of the brain in children investigated for developmental delay: what is the most appropriate imaging strategy? Eur Radiol 2011;21(09):1820-1830

17 Moeschler JB, Shevell M. Committee on Genetics. Comprehensive evaluation of the child with intellectual disability or global developmental delays. Pediatrics 2014;134(03):e903-e918

18 Spencer MD, Gibson RJ, Moorhead TW, et al. Qualitative assessment of brain anomalies in adolescents with mental retardation. AJNR Am J Neuroradiol 2005;26(10):2691-2697

19 Hart AR, Batty R, Widjaja E, et al. MRI in children with global developmental delay - a retrospective case note review. J Pediatr Neurol 2015;9:15-21

20 Verbruggen KT, Meiners LC, Sijens PE, Lunsing RJ, van Spronsen FJ, Brouwer OF. Magnetic resonance imaging and proton magnetic resonance spectroscopy of the brain in the diagnostic evaluation of developmental delay. Eur J Paediatr Neurol 2009;13(02):181-190

21 Mannerkoski M, Heiskala H, Raininko R, et al. Brain magnetic resonance imaging of siblings from families with two or more children with learning or intellectual disabilities and need for full-time special education. Acta Radiol 2009;50(04):437-445

22 des Portes V, Abaoub L, Joannard A, et al. So-called 'cryptogenic' partial seizures resulting from a subtle cortical dysgenesis due to a doublecortin gene mutation. Seizure 2002;11(04):273-277

23 Foerster BR, Ksar J, Petrou M, et al. Value of gadolinium in brain MRI examinations for developmental delay. Pediatr Neurol 2006; 35(02):126-130

24 Widjaja E, Nilsson D, Blaser S, Raybaud C. White matter abnormalities in children with idiopathic developmental delay. Acta Radiol 2008;49(05):589-595

25 Gabrielli O, Coppa GV, Manzoni M, et al. Minor cerebral alterations observed by magnetic resonance imaging in syndromic children with mental retardation. Eur J Radiol 1998;27(02): 139-144

26 Dangouloff-Ros V, Roux CJ, Boulouis G, et al. Incidental brain MRI findings in children: a systematic review and meta-analysis. AJNR Am J Neuroradiol 2019;40(11):1818-1823

27 Shevell MI, Majnemer A, Rosenbaum P, Abrahamowicz M. Etiologic yield of subspecialists' evaluation of young children with global developmental delay. J Pediatr 2000;136(05):593-598

28 Reid SM, Meehan EM, Arnup SJ, Reddihough DS. Intellectual disability in cerebral palsy: a population-based retrospective study. Dev Med Child Neurol 2018;60(07):687-694

29 Momen AA, Jelodar G, Dehdashti H. Brain magnetic resonance imaging findings in developmentally delayed children. Int J Pediatr 2011;2011:386984

30 Cauldwell C. Anesthesia risks associated with pediatric imaging. Pediatr Radiol 2011;41(08):949-950 